\title{
The Effect of Odour Valence and Odour Detection Threshold on the Withholding and Cancellation of Reach-to-Press Responses
}

\author{
Javier Albayay ${ }^{1}$ (D) . Umberto Castiello ${ }^{1} \cdot$ Valentina Parma $^{2,3}$
}

Received: 10 February 2021 / Accepted: 6 September 2021 / Published online: 27 September 2021

(c) The Author(s) 2021

\begin{abstract}
Introduction Withholding uninitiated actions and cancelling ongoing ones are two main components of response inhibition, a key element of the executive control. Inhibitory performance is sensitive to emotional contexts elicited by subliminal and supraliminal visual material. However, whether stimuli from other sensory modalities, such as odours, would equally modulate response inhibition remains unclear. Here, we aimed to assess the effect of task-irrelevant odours as a function of their valence and threshold on both action withholding and action cancellation of reach-to-press movements.

Method Thirty-two healthy participants performed a Go/No-Go task that included the presentation of pleasant (orange) and unpleasant (trimethyloxazole) odour primes at supra- and sub-threshold levels; clean air was included as a control condition. The reach-to-press responses were composed of an initial release phase and a subsequent reaching phase.

Results Only the supra-threshold pleasant (vs. control) odour impaired action withholding. Moreover, the pleasant (vs. control) odour-presented at both sub- and supra-threshold levels-elicited more accurate Go responses, whereas the sub- and supra-threshold pleasant and unpleasant (vs. control) odours triggered faster responses in the release phase. Additionally, only the supra-threshold pleasant (vs. unpleasant) odour impaired action cancellation in the reaching phase. Furthermore, reaching responses were slower following the supra-threshold unpleasant (vs. control) odour.

Conclusions Our findings extend the sparse literature on the impact of odour stimuli on goal-directed behaviour, highlighting the role of both odour valence and threshold in the modulation of response inhibition.

Implications Determining the mechanisms by which odour stimuli modulate response inhibition lays the foundations for research on odour-triggered disinhibition.
\end{abstract}

Keywords Response inhibition $\cdot$ Response readiness $\cdot$ Olfaction $\cdot$ Emotional priming $\cdot$ Reach-to-press

\section{Introduction}

Halting uninitiated and already initiated responses are central aspects of response inhibition (Aron 2011; Verbruggen and Logan 2017), a core component of executive control which allows people to implement adaptive goal-directed behaviours (Diamond 2013). Executive control is activated in a flexible manner and, as a result, inhibiting motor

Javier Albayay

javier.albayay@gmail.com

1 Department of General Psychology, University of Padova, Via Venezia 8, 35131 Padova, Italy

2 Department of Psychology, Temple University, 1701 N 13th St, Philadelphia, PA 19122, USA

3 Neuroscience Area, International School for Advanced Studies, Via Bonomea 265, 34151 Trieste, Italy responses is not an all-or-none process (Nguyen et al. 2016). Most of the studies on response inhibition have been focused on the withholding or cancellation of overt and covert (e.g. potential actions not overtly performed; Angelini et al. 2015, 2016) responses. Actions can be inhibited right before the initiation of the motor response (i.e. action withholding), after it has already started (i.e. action cancellation), or they cannot be withheld and the erroneously planned action is implemented to completion (i.e., unsuccessful inhibition; Nguyen et al. 2016). Furthermore, and of relevance to the present study, inhibitory processes can be at work even when actions are triggered at the subliminal level by sensory inputs and emotionally laden stimuli (Parkinson et al. 2017).

In this respect, a number of studies have shown that response inhibition can be triggered by subliminal (nonemotional) visual stimuli in masked prime paradigms (Boy et al. 2008; Chiu and Aron 2014; Eimer and Schlaghecken 
2002; Hughes et al. 2009; Parkinson and Haggard 2014; Sumner 2007; van Gaal et al. 2009, 2010). For instance, Hughes et al. (2009) found that task-irrelevant masked Go (vs. No-Go and neutral) primes facilitated response readiness (faster reaction time, RT) and impaired response inhibition (higher rates of unsuccessful withholding). The opposite pattern was found for No-Go (vs. neutral) primes. In a similar vein, van Gaal et al. (2010) found that varying the strength of the masking procedure for primes-from weak (i.e. accessing conscious awareness) to strong (i.e. lack of conscious awareness) — slows down response readiness only when inhibition is triggered by strongly masked No-Go (vs. Go) targets.

The effect of subliminal priming on motor inhibition has not only been uncovered via simple keypress/release tasks, such as classical Go/No-Go paradigms, but also via tasks that require more complex action planning and execution (Ocampo and Finkbeiner 2013; Ocampo et al. 2015). In demanding situations, movement complexity affects the effectiveness of response inhibition (Gálvez-García et al. 2018). In line with this idea, Ocampo et al. (2015) found that participants can use non-consciously perceived visual information to modify ongoing arm reaching movements. The movement time and maximum velocity of correct reaching responses were faster in congruent trials (i.e. primes and targets shared features, for instance, the same letter), whereas action cancellation was less successful in incongruent trials. In other words, there is evidence that subliminal visual stimuli can modulate the inhibition of ongoing actions.

In contrast to the wealth of studies on subliminal nonemotional visual stimuli, there is a paucity of evidence on the inhibitory effects dictated by emotional stimuli presented non-consciously. In a variation of the Go/No-Go task, Parkinson et al. (2017) found that subliminally presented angry (vs. happy, fear and neutral) faces increased the willingness to inhibit motor responses in free-choice trials. However, the effect disappears when faces were presented supraliminally. The authors concluded that the subliminal perception of anger specifically serves as a non-conscious cue that triggers an adaptive conscious withholding process. These results suggest that the effect of visually presented subliminal emotional stimuli on response inhibition might be emotion-specific.

On the basis of the available evidence, two aspects remain understudied: (i) whether the cancellation of an ongoing action is modulated by emotional non-conscious stimuli; (ii) what are the effects of subliminal and supraliminal emotional stimuli on response inhibition. Here, we try to fill these gaps by investigating how odours-stimuli that constitute powerful emotional triggers, both when presented above and below the level of conscious awareness-affect two forms of response inhibition: action withholding and action cancellation. In virtue of their specific features, supported by their unique neural organisation (Wilson and Stevenson 2006), their effectiveness as affective primes (Smeets and Dijksterhuis 2014), their power as strong emotional (Adolph and Pause 2012) and automatic action triggers (Castiello et al. 2006), odours offer a promising possibility to test the boundaries of emotional human response inhibition.

The aim of this study was to determine whether taskirrelevant odours would modulate response inhibition in a modified version of the Go/No-Go task (see "Methods" section below). To this end, we presented healthy participants with sub- and supra-threshold pleasant (orange) and unpleasant (trimethyloxazole; a burnt nutty scent) odour primes in two separate experimental sessions. Unlike most of Go/No-Go paradigms in which participants are requested to respond by simple motor actions (e.g. keypress or key release; Albayay et al. 2019; Chiu et al. 2014; Parkinson and Haggard 2014; van Gaal et al. 2010), here the participants responded by performing a more complex reach-to-press action (e.g. Chen and Saunders 2018; Gálvez-García et al. 2018). This movement was composed of an initial release phase (i.e. releasing the dominant hand from a switch) and a subsequent reaching phase (i.e. reaching and pressing a keyboard's spacebar). From the release phase, we measured the rate of unsuccessfully withheld No-Go responses, the rate of omitted Go responses, and the RT of correct Go responses. As for the reaching phase, we measured the rate of unsuccessfully cancelled No-Go responses and the movement time (MT) of correct Go responses. In line with previous findings (Albayay et al. 2019), when supra-threshold odours are presented, we anticipate higher rates of unsuccessful withholding when a pleasant odour is delivered, given that more inhibitory resources are required as compared to the control conditions (Chiu et al. 2014). We also expect a lower rate of omissions to Go and faster RT when a pleasant odour prime is presented, under the assumption that pleasant contexts bias approach behaviours (Chiu et al. 2014; Waring et al. 2019). As for the reaching phase, analogously to the interference effects found by Ocampo et al. (2015), we considered the pleasant and unpleasant primes as Go and No-Go primes, respectively. Thus, we anticipate higher rates of unsuccessful cancellation when a pleasant odour (Go prime) is delivered and slower MT when an unpleasant (No-Go prime) odour is presented. Despite the evidence on the modulation of inhibitory performance by subliminal visual stimuli in nonemotional masked prime (e.g. Hughes et al. 2009; Parkinson and Haggard 2014; Sumner, 2007; van Gaal et al. 2009, 2010) and free-choice paradigms (Parkinson et al. 2017), we expect that sub-threshold odour primes-which can be considered less arousing if non-threatening (Bensafi et al. 2002; Li 2014) - will not significantly modulate response inhibition, in line with previous studies showing that particularly high arousing affective stimuli impair response inhibition (Pearlstein et al. 2019; Verbruggen and De Houwer, 2007). 
Indeed, odours are more likely to attract attention when they are particularly pleasant, strong and when they signal danger or contamination (Smeets and Dijksterhuis 2014). In addition, we can expect the sub-threshold odours to lose their valence effect; greater pleasantness is modulated by odour intensity in a U-shaped manner when presented at detectable concentrations (Moskowitz et al. 1976; Rouby et al. 2009).

\section{Methods}

\section{Participants}

Thirty-two healthy participants aged between 21 and 27 (mean age $=23.4 \pm 1.5$ years old, 15 women) were recruited by convenience sampling. An a priori power analysis ( $\mathrm{G}^{*}$ Power; Faul et al. 2007) suggested a sample size of twenty-nine participants for a medium effect size ( $F$ test family, ANOVA: repeated measures, within factors) at power $=0.9$ and $\alpha=0.05$. This sample size is greater than earlier behavioural research on the impact of affective stimuli on response inhibition. For instance, samples ranged from 18 to 25 in the experiments of Chiu et al. (2014), Verbruggen and De Houwer (2007), and Yang et al. (2014). Participants were selected based on an online survey that accounted for the following exclusion criteria: past head trauma, current smokers or smokers until 6 months ago, habitual drinkers (more than five times a week), anxiety (score $<43$ at the State-Trait Inventory for Cognitive and Somatic Anxiety; Grös et al. 2007), and depression (score $<17$ at the Beck Depression Inventory-II; Beck et al. 1996). All participants were right-handed (score $>60$ at the Edinburgh Handedness Inventory; Oldfield 1971), normosmic (score $\geq 10$ at the Identification subtest of the Sniffin' Sticks test; Burghart ${ }^{\circledR}$, Wedel, Germany; Hummel et al. 2007), and self-reported normal or corrected to normal vision. Participants were instructed to avoid eating or drinking anything but water from one hour prior to each experimental session and to avoid wearing any scented products. All participants gave written informed consent and did not receive any monetary compensation. The study was approved by the local Institutional Review Board (name of the institution) and was conducted in accordance with the Declaration of Helsinki (World Medical Association 2013) and the ethical standards of the American Psychological Association (2017).

\section{Apparatus and Stimuli}

\section{Odour Stimuli}

Odour solutions were presented at supra- and sub-threshold levels during the experiment. Supra-threshold stimuli consisted of two odours diluted with propylene glycol: orange
(30\% v/v, Givaudan), and 2,4,5-trimethyloxazole $(0.5 \%$ $\mathrm{v} / \mathrm{v}$, Sigma-Aldrich; a burnt nutty scent). We selected these concentrations based on previous research showing that the orange and the trimethyloxazole odours are isointense and different in pleasantness. Following Albayay et al. (2019), we anticipated the orange odour to be perceived as pleasant and the trimethyloxazole odour as unpleasant. Sub-threshold stimuli corresponded to variable dilutions of the suprathreshold solutions. Following a single-staircase procedure (Hummel et al. 1997; Li et al. 2007), participant-specific thresholds were estimated by presenting 12 dilutions of each odour. The supra-threshold stimuli corresponded to the dilution starting point for each odour. From these initial stimuli, both odours were diluted geometrically by creating $2 \mathrm{~mL}$ consecutive solutions in which one was half of the previous one (end concentration for orange $=0.014648438 \% \mathrm{v} / \mathrm{v}$, end concentration for trimethyloxazole $=0.000244141 \%$ $\mathrm{v} / \mathrm{v})$. These dilutions were stored in sanitised $100 \mathrm{~mL}$ amber bottles. Participants were presented with a specific dilution along with two jars containing propylene glycol $(2 \mathrm{~mL})$ and were asked to identify the jar containing the odorant. The criterion from the threshold subtest of the Sniffin' Sticks test (Burghart®, Wedel, Germany; Hummel et al. 2007) was used to obtain participant's thresholds (for a detailed description of the procedure we refer the interested reader to Hummel et al. 1997). Following Li et al. (2007), the subthreshold odours used in the experiment were concentrations two dilutions lower than the measured participant's thresholds. Clean air (over propylene glycol) was used as a control condition against both supra- and sub-threshold odours. The odour solutions used in the experiment were stored in sanitised glass jars ( $3 \mathrm{~mL}$ solution in straight-sided glass $4 \mathrm{oz}$ jars, Uline, Pleasant Prairie, WI, USA), and were delivered via a customised computer-controlled olfactometer (Sniff-0, CyNexo, Udine, Italy, http://www.cynexo.com). Odour stimuli were delivered at a flow of $3 \mathrm{~L} / \mathrm{min}$ whereas a constant flowing air stream of $0.5 \mathrm{~L} / \mathrm{min}$ remained constant thought the experiment; odours stimuli were delivered via cannulas covered with custom-made nose-pieces birhinally placed in the participants' nostrils (Parma et al. 2015).

\section{Visual Stimuli}

Go/No-Go visual targets (a white circle and a white square, $2.5 \times 2.5 \mathrm{~cm})$, fixation cross $(1.5 \times 1.5 \mathrm{~cm})$ and textual information were presented on a 19" LCD monitor (NEC AccuSync LCD93VM, $1280 \times 1024$ resolution at $60 \mathrm{~Hz}$ ) against a black background.

\section{Experimental Setup}

During the main task, participants placed their right hand on a starting switch $(10 \times 7 \mathrm{~cm})$ positioned on a $90 \times 90-\mathrm{cm}$ 
table at a 23-cm distance from the edge of the table and aligned with the participant's body midline axis. A computer keyboard $(30 \times 10 \mathrm{~cm})$ was placed at $20 \mathrm{~cm}$ from the switch (50 $\mathrm{cm}$ from the edge of the table). The LCD monitor was placed at $5 \mathrm{~cm}$ from the keyboard $(65 \mathrm{~cm}$ from the edge of the table). During the experimental tasks, the participants rested their head on a chin rest (see Fig. 1a).

\section{Procedure}

\section{Go/No-Go Task}

Participants performed a modified version of the Go/No-Go task (Donders 1969; see Fig. 1b) that included the delivery of task-irrelevant odour primes. Each trial started with a black screen and the delivery of clean air for $500 \mathrm{~ms}$. Then, a white fixation cross (i.e. sniff cue) appeared in the centre of the screen as one of the three odour stimuli was delivered for $1 \mathrm{~s}$ (time enough for the detection of an odour in a single sniff; > 750 ms reported by Gottfried et al. 2002); clean air was disabled during the delivery of an odour. After this, the target (a circle or a square) replaced the fixation cross and clean air was delivered once again. The target remained on screen for $500 \mathrm{~ms}$ regardless of the participant's response. The intertrial interval (ITI) ranged from 400 to $600 \mathrm{~ms}$ (with an average of $500 \mathrm{~ms}$ ). During the ITI, the screen remained black. Clean air was delivered during the ITI to clean any residuals of previously presented odours. Each trial lasted on average $2.5 \mathrm{~s}$. At the beginning of the task, participants were instructed to place the ulnar side of the right hand on a customised starting switch with the tips of the index and the thumb touching each other. When the Go target (circle) was presented, participants were instructed to respond as fast as possible by releasing the starting switch and then pressing the spacebar of the keyboard with their right index finger. Participants were instructed to release the starting switch whilst the target was on screen (500 ms response deadline). After responding, participants moved their right hand back to the initial position on the switch. When the No-Go target (square) was presented, participants were asked to hold their hand on the starting switch until the next trial. Half of the sample followed the opposite mapping rule (i.e. square as Go target and circle as No-Go target). In other words, the switch activity provided the measure of release vs. withholding, and the space bar activity provided the measure of reaching vs cancellation (see Fig. 1c). Finally, the rate a

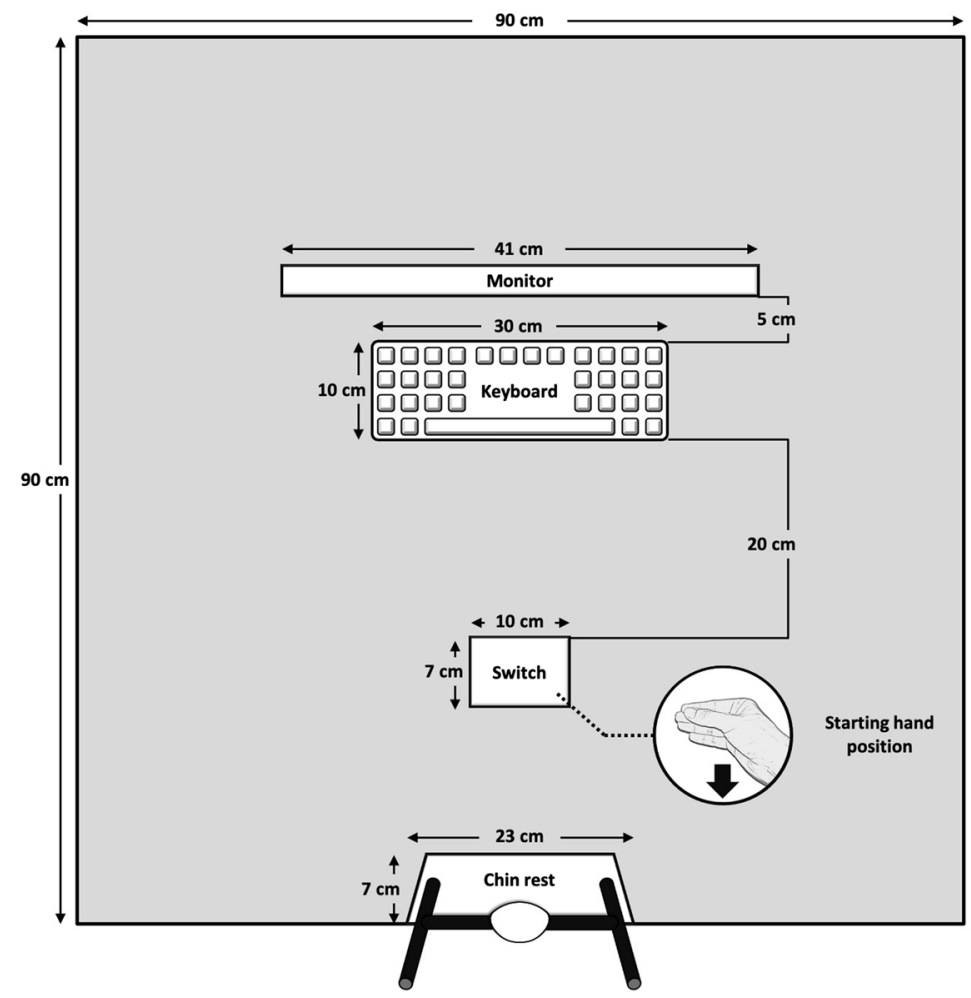

b

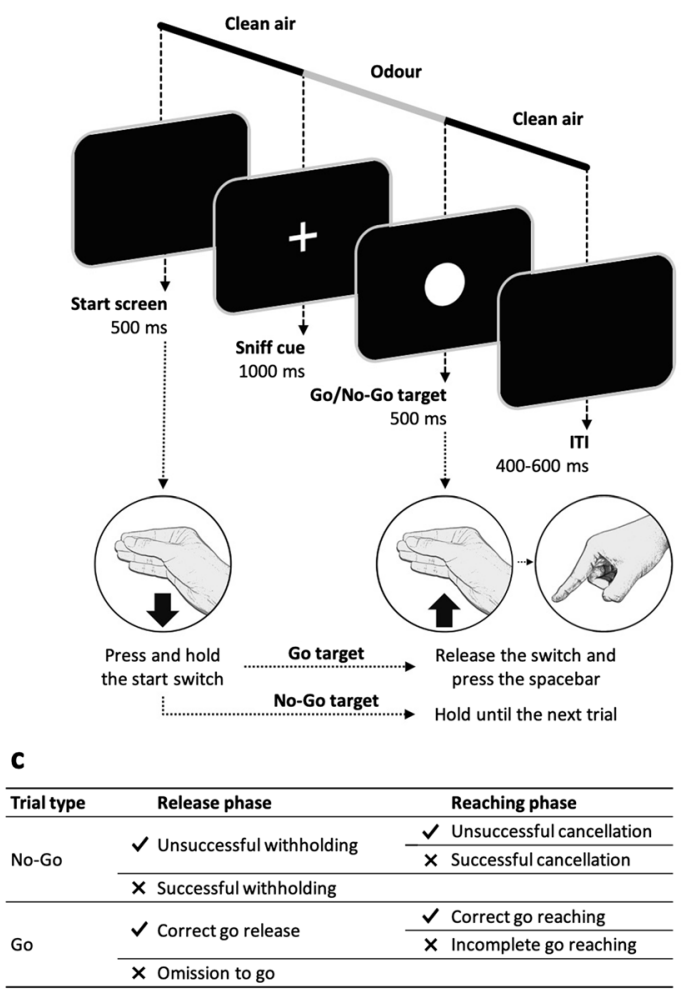

Fig. 1 a Experimental setup. b Graphical representation of the Go/ No-Go task; clean air was delivered uninterruptedly in control trials. c Possible outcomes for the release and the reaching phases of the motor response in Go and No-Go trials; incomplete Go reaching responses were not considered for analysis. The tick mark indicates executed release or reaching action, whereas the $\times$ indicates non-executed release or reaching action 
of failed responses and mean RT were presented for $10 \mathrm{~s}$ as feedback on screen at the end of each block. This allowed the experimenter to monitor the performance of the participants during the experimental sessions.

The experiment was composed of two sessions, in which only supra- or sub-threshold odours were presented; sessions were 7-10 days apart (mean $=7.8 \pm 1.0$ days). The order of the session was counterbalanced. In addition, the mapping instructions of the Go/No-Go targets were opposite on each session to minimise learning effects (e.g. for a given participant the circle served as Go target in the first session and as No-Go target in the second session). Given that neither the order of the sessions nor the mapping instructions had a significant effect on the dependent variables we collapsed the data from all participants. On each session, the Go/No-Go task was composed of five blocks. Each block included 120 trials (40 per odour stimuli) from which $20 \%$ were No-Go trials (Wessel 2017). Trials were presented following a pseudo-randomised order where No-Go trials were equiprobably preceded by 1, 3, 5 or 7 Go trials (Zamorano et al. 2014). A given odour stimulus was never presented twice consecutively in order to avoid habituation effects; constant changes in the olfactory scenario increase the probability of detecting the odour stimuli at short ITI (Croy et al. 2015). It should be noted that four participants reported having perceived an odour in some trials in the sub-threshold session. However, they were not able to identify the odours. Further, when presented with the corresponding concentration two dilutions lower than the subjectively determined sub-threshold levels (following Hummel et al. 1997; Li et al. 2007), none of the participants was able to distinguish between the sub-threshold odours and no-odour.

\section{Breathing Training}

At the beginning of each session, participants performed a breathing training which consisted of 30 trials following the same sequence of the Go/No-Go trials. The only difference refers to the fact that clean air was always presented, and no action was required following the presentation of $\mathrm{Go} / \mathrm{No}-\mathrm{Go}$ targets. Participants were instructed to sniff once when the fixation cross (sniff cue) appeared on the centre of the screen and then exhale until the end of the trial. The experimenter visually examined if the participants breathed as instructed and debriefed them at the end of the training.

\section{Familiarisation Block}

On each session (after the breathing training) participants performed a Go/No-Go familiarisation block composed of 30 trials identical to those of the main task. Only 6/30 trials included the presentation of an odour. This was done to avoid overexposure to the odour stimuli and adaptation during the familiarisation block. Participants proceeded to the main task if they reached or exceeded $85 \%$ accuracy in the familiarisation block.

\section{Rating Procedure}

At the end of each experimental block, participants were asked to rate the pleasantness and intensity of each odour stimulus on visual analogue scales (VAS) ranging from 0 (not at all) to 100 (very much). This procedure was only performed in the session where supra-threshold odours were presented. Participants had a 1-min rest period before starting a new block. The experimenter reminded the participants to breathe as trained and to respond as accurately and as fast as possible when prompted. The experiment was carried out using the E-Prime 2.0 software (Psychology Software Tools, Inc. 2012). Each experimental session lasted about $60 \mathrm{~min}$.

\section{Statistical Analyses}

From the rating procedures of the supra-threshold condition, the perceived pleasantness and intensity of the odour stimuli (i.e. VAS scores) were considered as dependent variables. Regarding the release phase of the response, the following dependent variables were considered: rate of unsuccessful withholding (i.e. trials in which the participants released the starting switch when a No-Go target was presented), rate of omissions to Go (i.e., trials in which the participants did not release the starting switch when a Go target was presented), and RT of correct Go release responses (i.e. time in ms elapsed from the onset of a Go target until the participant released the starting switch). As for the reaching phase of the response, the following dependent variables were considered for the analyses: rate of unsuccessful cancellation (i.e. trials in which the participants reached and pressed the space bar after releasing the starting switch when a No-Go target was presented) and MT of correct Go reaching responses (i.e. time in ms elapsed from the switch release action until the participant pressed the spacebar when a Go target was presented).

All analyses were performed using RStudio (version 1.1.383; RStudio Team 2016). Linear mixed-effects models (LME) were computed for the perceived odour intensity and pleasantness, and the RT and MT of correct Go trials. Generalised linear mixed-effects models (GLME) with binomial link function were computed for the rates of unsuccessful withholding, omissions to Go, and unsuccessful cancellation. LME and GLME were computed using the nlme (Pinheiro et al. 2020) and the lme4 (Bates et al. 2018) packages. Correct Go release responses exceeding 2.5 standard deviations were removed ( $2.6 \%$ of the data). Odour intensity and pleasantness models included the factors odour (clean air vs. orange vs. trimethyloxazole) and cycle (moments when 
participants rated the odour stimuli) as fixed effects. For all the remaining models the factors valence (control vs. pleasant vs. unpleasant) and threshold (supra- vs. sub-threshold) were included as fixed effects. All the LME and GLME models included participants as a random effect in order to account for the stochastic variability in the data and reflect a more general estimate of the fixed effects (Singmann and Kellen 2017).

We compared the computed models for a given dependent variable following a model comparison approach (McElreath 2016) and contrasted them using the Akaike information criterion (AIC), considering the model with the lowest AIC as the best fitting model (Vieno et al. 2018). The models including an interaction term were contrasted against models that included main effects but not the interaction (e.g. Model A $=\mathrm{RT} \sim$ valence $\times$ threshold vs. Model $\mathrm{B}=\mathrm{RT} \sim$ valence + threshold). Furthermore, the models containing only one main effect were contrasted against models containing the two main effects (e.g., Model B vs. Model $\mathrm{C}=\mathrm{RT} \sim$ valence, and Model B vs. Model $\mathrm{D}=\mathrm{RT} \sim$ threshold). We computed the exponent of the difference between the AIC of the models to determine the relative likelihood of a given model $\left[\mathrm{AIC}_{\mathrm{RL}}=\exp (\Delta \mathrm{AIC} / 2)\right] ; p$ values were obtained from likelihood ratio tests. We used the lsmeans package (Lenth 2016), selecting the Tukey method for the adjustment of $p$ values, to perform planned contrasts on the significant effects and, particularly, to compare the odour conditions within the supra- and sub-threshold conditions. Furthermore, we estimated the marginal and conditional $R^{2}$ from the piecewiseSEM package (Lefcheck 2016) to account for the proportion of variance explained by the fixed effects (marginal; $R_{\mathrm{m}}^{2}$ ) and by both the fixed and random effects (conditional; $R_{\mathrm{c}}^{2}$ ). For example, in Model A (described above), the $R_{\mathrm{m}}^{2}$ and the $R^{2}$ correspond to the variance explained by the interaction valence $\times$ threshold, and by this interaction plus the participants' random effect, respectively. We estimated and reported the mean and standard deviation of each condition for LME models and the percent of failed trials of each condition for GLME model.

\section{Results}

\section{Odours are Isointense and Differ in Pleasantness}

A significant main effect of odour was observed on the perceived intensity of the odour stimuli, $\chi^{2}(2)=557.16$, $p<0.001, \mathrm{AIC}_{\mathrm{RL}}>100, R_{\mathrm{m}}^{2}=0.625, R_{\mathrm{c}}^{2}=0.755$, whereas the main effect of cycle, $\chi^{2}(4)=5.075, p=0.280$, $\mathrm{AIC}_{\mathrm{RL}}=0.232, R_{\mathrm{m}}^{2}=0.003, R_{\mathrm{c}}^{2}=0.089$, and the interaction odour $\times$ cycle, $\chi^{2}(8)=5.094, p=0.747, \mathrm{AIC}_{\mathrm{RL}}=0.004$, $R_{\mathrm{m}}^{2}=0.631, R_{\mathrm{c}}^{2}=0.761$, were not significant. Clean air $(8.2 \pm 14.4)$ was rated as less intense than both the orange
$(70.1 \pm 23.9, p<0.001)$ and trimethyloxazole $(66.8 \pm 26.1$, $p<0.001)$ odours. As expected, the perceived intensity of the orange and trimethyloxazole odours did not differ significantly $(p=0.236)$. The main effect of odour on the perceived pleasantness was also significant, $\chi^{2}(2)=427.43$, $p<0.001, \mathrm{AIC}_{\mathrm{RL}}>100, R_{\mathrm{m}}^{2}=0.551, R_{\mathrm{c}}^{2}=0.666$, while the main effect of cycle, $\chi^{2}(4)=0.448, p=0.978, \mathrm{AIC}_{\mathrm{RL}}=0.023$, $R_{\mathrm{m}}^{2}<0.001, R_{\mathrm{c}}^{2}=0.076$, and the interaction odour $\times$ cycle, $\chi^{2}(8)=9.304, p=0.317, \mathrm{AIC}_{\mathrm{RL}}=0.035, R_{\mathrm{m}}^{2}=0.558$, $R^{2}{ }_{\mathrm{c}}=0.674$, were not. The orange odour $(77.8 \pm 17.3)$ was rated as more pleasant than the trimethyloxazole odour $(27.7 \pm 24.2, p<0.001)$ and clean air $(44.7 \pm 13.4$, $p<0.001)$. Further, the trimethyloxazole odour was rated as more unpleasant than clean air $(p<0.001)$. The average response time of the rating procedures was $1861 \pm 1115 \mathrm{~ms}$. Given that the factor cycle did not show any significant effect, we contend that both odour pleasantness and intensity remained constant throughout the experiment.

\section{Release Phase}

\section{Supra-threshold Pleasant (vs. Control) Odours Impair Action Withholding}

The main effect of valence on the rate of unsuccessful withholding was not significant, $\chi^{2}(2)=3.210, p=0.201$, $\mathrm{AIC}_{\mathrm{RL}}=0.674, R_{\mathrm{m}}^{2}<0.001, R_{\mathrm{c}}^{2}=0.215($ control $=31.5 \%$, pleasant $=33.7 \%$, unpleasant $=32.2 \%)$. On the other hand, a significant main effect of threshold was retrieved, $\chi^{2}(1)=22.023, p<0.001, \mathrm{AIC}_{\mathrm{RL}}>100, R_{\mathrm{m}}^{2}=0.003$, $R^{2}{ }_{\mathrm{c}}=0.218$ (sub-threshold $=30.4 \%$, supra-threshold $=34.6 \%$ ). The interaction valence $\times$ threshold reached significance too, $\chi^{2}(2)=7.457, p=0.024, \mathrm{AIC}_{\mathrm{RL}}=5.631$, $R_{\mathrm{m}}^{2}=0.004, R_{\mathrm{c}}^{2}=0.220$. Planned contrasts (see Fig. 2a, b) revealed that more errors were elicited when presented with the supra-threshold pleasant odour (37.3\%) as compared the control odour $(31.7 \%, p=0.024)$, whereas other comparisons were not significant both in the supra-threshold condition [control vs. unpleasant $(34.8 \%, p=0.473)$, pleasant vs. unpleasant $(p=0.782)]$ and in the sub-threshold condition [control (31.3\%) vs. pleasant $(30.3 \%, p=0.990)$, control vs. unpleasant $(29.7 \%, p=0.920)$, pleasant vs. unpleasant $(p=0.999)]$. In short, action withholding of the initial release phase of a reach-to-press response was impaired when smelling a supra-threshold pleasant odour as compared to when no odour was smelled.

\section{Decrease in Omissions to Go When Exposed to Sub- and Supra-threshold Pleasant (vs. Control) Odours}

A significant main effect of valence was found on the rate of omissions to Go, $\chi^{2}(2)=17.382, p<0.001, \mathrm{AIC}_{\mathrm{RL}}>100$, $R_{\mathrm{m}}^{2}=0.001, R_{\mathrm{c}}^{2}=0.091$, where more errors were committed 
Fig. 2 Left panel: Results of the sub-threshold condition per odour valence. a Rate of unsuccessful withholding. c Rate of omissions to Go. e Reaction time of correct Go release responses. Right panel: Results of the supra-threshold condition per odour valence. b Rate of unsuccessful withholding. d Rate of omissions to Go f Reaction time of correct Go release responses. Error bars represent standard error of the mean

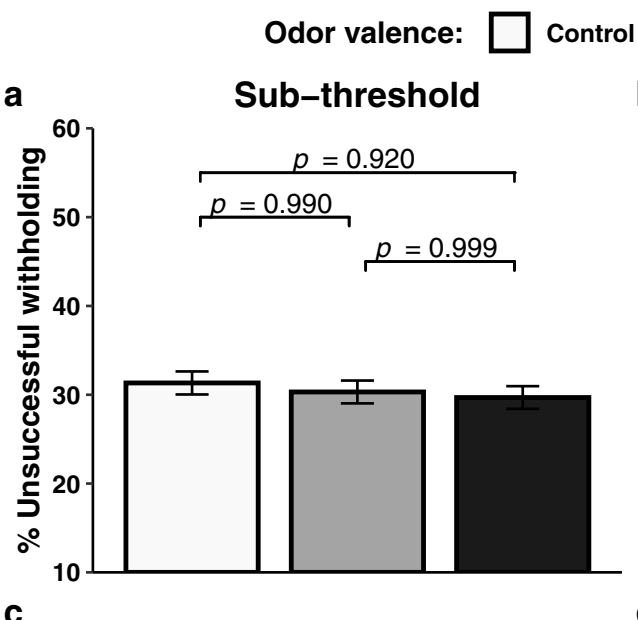

C
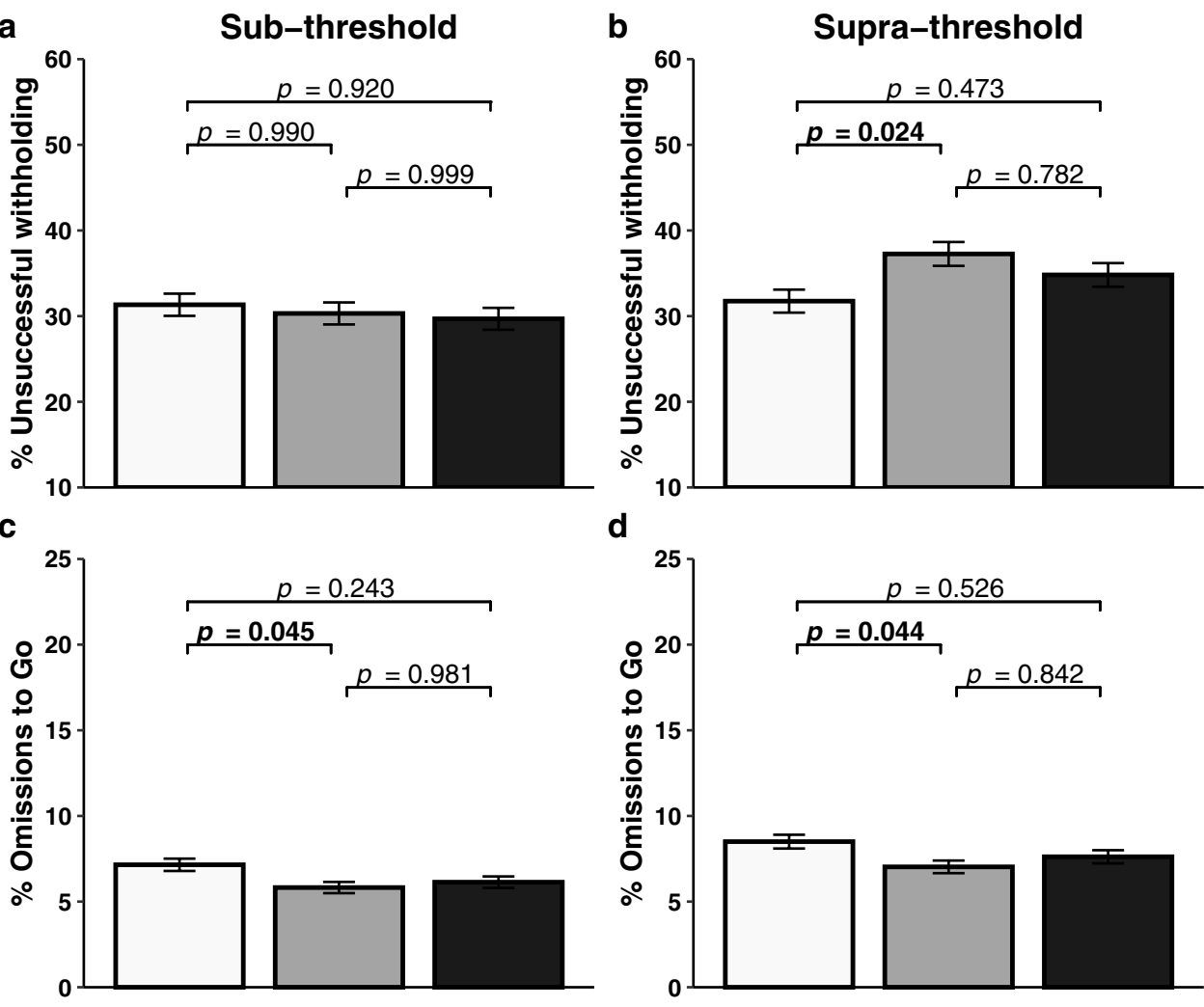

d
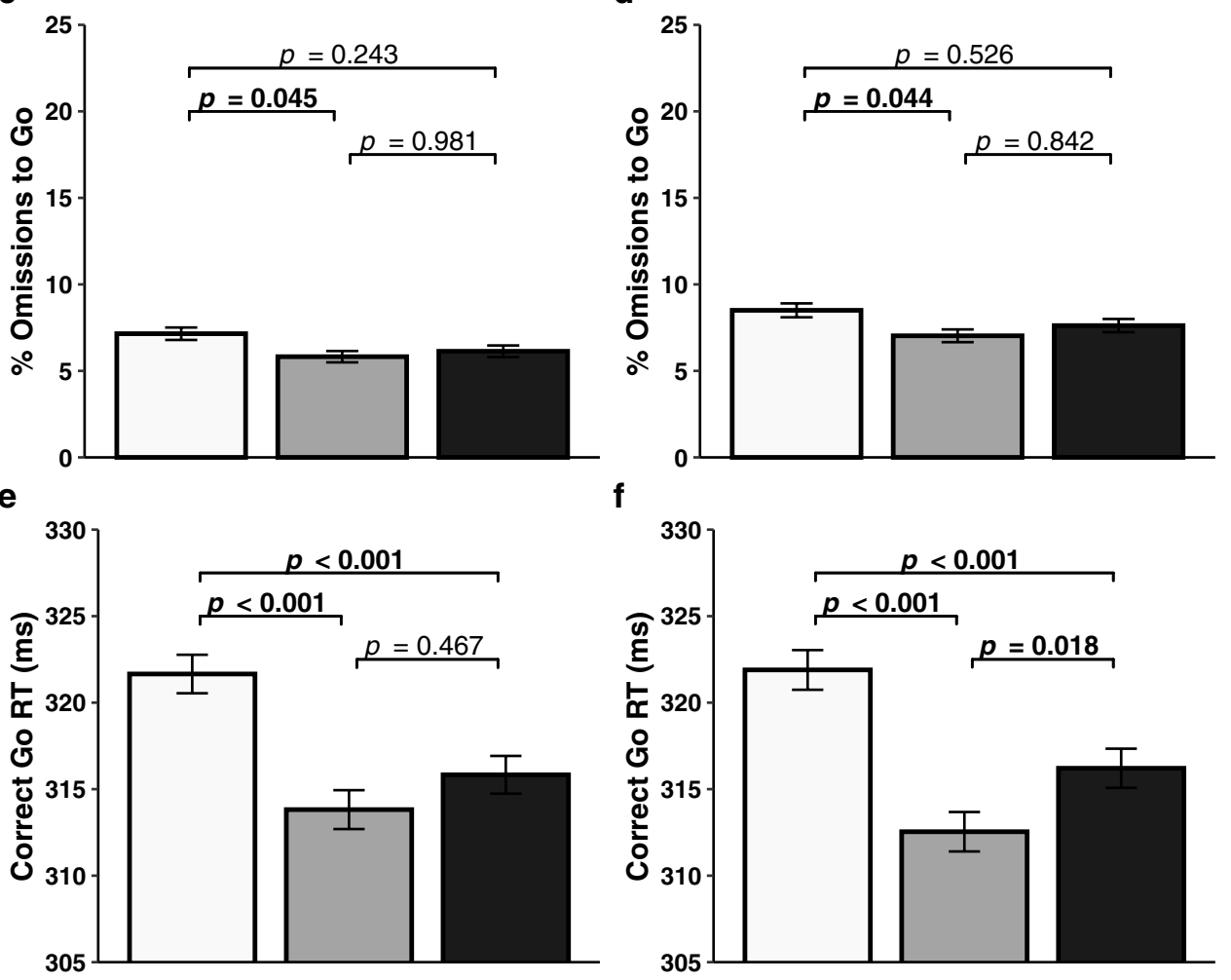

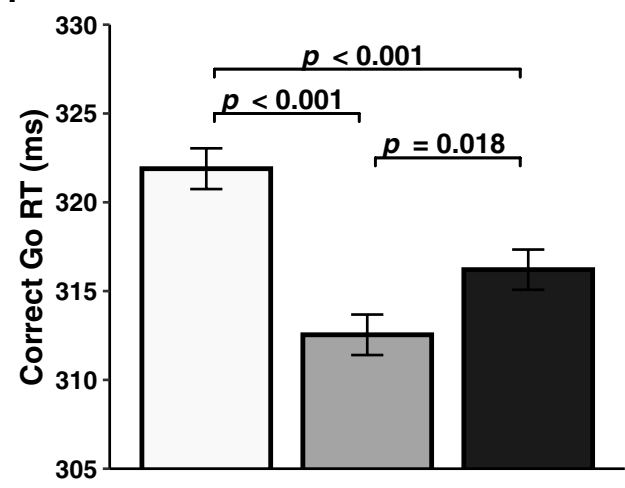

during exposure to the control odour (7.8\%) as compared to pleasant $(6.4 \%, p<0.001)$ and unpleasant odours $(6.8 \%$, $p=0.017$ ), while no differences were found between the pleasant and the unpleasant odours $(p=0.369)$. The main effect of threshold was significant too, $\chi^{2}(1)=35.359, p<0.001$, $\mathrm{AIC}_{\mathrm{RL}}>100, R_{\mathrm{m}}^{2}=0.001, R_{\mathrm{c}}^{2}=0.093$, with more errors in the supra-threshold condition $(7.7 \%)$ than in the sub-threshold one $(6.4 \%)$. The interaction valence $\times$ threshold did not reach significance, $\chi^{2}(2)=0.175, p=0.916, \mathrm{AIC}_{\mathrm{RL}}=0.148$, $R_{\mathrm{m}}^{2}=0.002, R_{\mathrm{c}}^{2}=0.093$. Planned contrasts (see Fig. $2 \mathrm{c}, \mathrm{d}$ ) showed that participants tended to make more errors during exposure to the control odour as compared to the pleasant odour in both sub-threshold $(7.1 \%$ vs. $5.8 \%, p=0.045)$ and supra-threshold ( $8.5 \%$ vs. $7.0 \%, p=0.044)$ conditions.
Other comparisons were not significant [sub-threshold: control vs. unpleasant $(6.1 \%, p=0.243)$, pleasant vs. unpleasant $(p=0.981)$; supra-threshold: control vs. unpleasant $(7.6 \%$, $p=0.526)$, pleasant vs. unpleasant $(p=0.842)]$. In brief, the participants were more likely to correctly release the switch in Go trials when they smelled the pleasant odour presented at both sub- and supra-threshold levels.

\section{Correct Go Release responses are Faster when Sub- and Supra-threshold Pleasant and Unpleasant (vs. Control) Odours are Presented}

A significant main effect of valence was revealed on the RT of correct Go release responses, $\chi^{2}(2)=143.92$, 
$p<0.001, \mathrm{AIC}_{\mathrm{RL}}>100, R_{\mathrm{m}}^{2}=0.002, R_{\mathrm{c}}^{2}=0.532$, with faster responses during exposure to both pleasant $(313 \pm 76 \mathrm{~ms})$ and unpleasant odours $(316 \pm 75 \mathrm{~ms})$ as compared to the control odour $(322 \pm 75 \mathrm{~ms}, p \mathrm{~s}<0.001)$, especially in pleasant vs. unpleasant $(p=0.001)$. Instead, neither the main effect of threshold, $\chi^{2}(1)=0.897$, $p=0.344, \mathrm{AIC}_{\mathrm{RL}}=0.576, R_{\mathrm{m}}^{2}<0.001, R_{\mathrm{c}}{ }_{\mathrm{c}}=0.529$ (subthreshold $=317 \pm 76 \mathrm{~ms}$, supra-threshold $=317 \pm 75 \mathrm{~ms}$ ), nor the interaction valence $\times$ threshold, $\chi^{2}(2)=1.129$, $p=0.569, \mathrm{AIC}_{\mathrm{RL}}=0.238, R_{\mathrm{m}}^{2}=0.002, R_{\mathrm{c}}^{2}=0.532$, were significant. Planned contrasts (see Fig. 2e, f) revealed a valence effect in the supra-threshold condition [control $(322 \pm 75 \mathrm{~ms})$ vs. pleasant $(312 \pm 75 \mathrm{~ms}, p<0.001)$, control vs. unpleasant $(316 \pm 74 \mathrm{~ms}, p<0.001)]$, with trials primed with the pleasant (vs. unpleasant) odour being faster $(p=0.018)$. In contrast, in the sub-threshold condition [control ( $322 \pm 76 \mathrm{~ms})$ vs. pleasant $(314 \pm 77 \mathrm{~ms}$, $p<0.001)$, control vs. unpleasant $(316 \pm 75 \mathrm{~ms}$, $p<0.001)$ ], no differences were found between trials preceded by pleasant and unpleasant odours $(p=0.467)$. To sum up, response readiness in the release phase was facilitated when smelling sub- and supra-threshold odour stimuli, especially when the supra-threshold pleasant odour was presented.

\section{Reaching Phase}

\section{Supra-threshold Pleasant (vs. Unpleasant) Odours Impair Action Cancellation}

The main effect of valence on the rate of unsuccessful cancellation was not significant, $\chi^{2}(2)=4.224, p=0.121$, $\mathrm{AIC}_{\mathrm{RL}}=1.118, R_{\mathrm{m}}^{2}=0.001, R_{\mathrm{c}}^{2}=0.136($ control $=10.2 \%$, pleasant $=9.8 \%$, unpleasant $=7.0 \%$ ). Conversely, a significant main effect of threshold was found, $\chi^{2}(1)=4.719$, $p=0.030, \mathrm{AIC}_{\mathrm{RL}}=3.895, R_{\mathrm{m}}^{2}=0.002, R_{\mathrm{c}}^{2}=0.138$ (subthreshold $=8.5 \%$, supra-threshold $=9.5 \%$ ). The interaction valence $\times$ threshold was significant too, $\chi^{2}(2)=7.685$, $p=0.021, \mathrm{AIC}_{\mathrm{RL}}=6.312, R_{\mathrm{m}}^{2}=0.006, R_{\mathrm{c}}^{2}=0.143$. Planned contrasts (see Fig. 3a, b) showed that participants had more difficulties to stop an ongoing inappropriate action when exposed to the pleasant odour $(12 \%)$ as compared to the unpleasant one $(6.0 \%, p=0.032)$ in the supra-threshold condition. No other significant comparison emerged [suprathreshold: control $(10.2 \%)$ vs. pleasant $(p=0.971)$, control vs. unpleasant $(p=0.252)$; sub-threshold: control $(10.2 \%)$ vs. pleasant $(7.2 \%, p=0.710)$, control vs. unpleasant $(8.2 \%$, $p=0.997)$, pleasant vs. unpleasant $(p=0.944)]$. In brief, cancelling an erroneously triggered response was more challenging when smelling the pleasant (vs. unpleasant) odour but only when presented at supra-threshold level.
Fig. 3 Left panel: Results of the sub-threshold condition per odour valence. a Rate of unsuccessful cancellation. c Movement time of correct Go reaching responses. Right panel: Results of the supra-threshold condition per odour valence. $\mathbf{b}$ Rate of unsuccessful cancellation. d Movement time of correct Go reaching responses. Error bars represent standard error of the mean
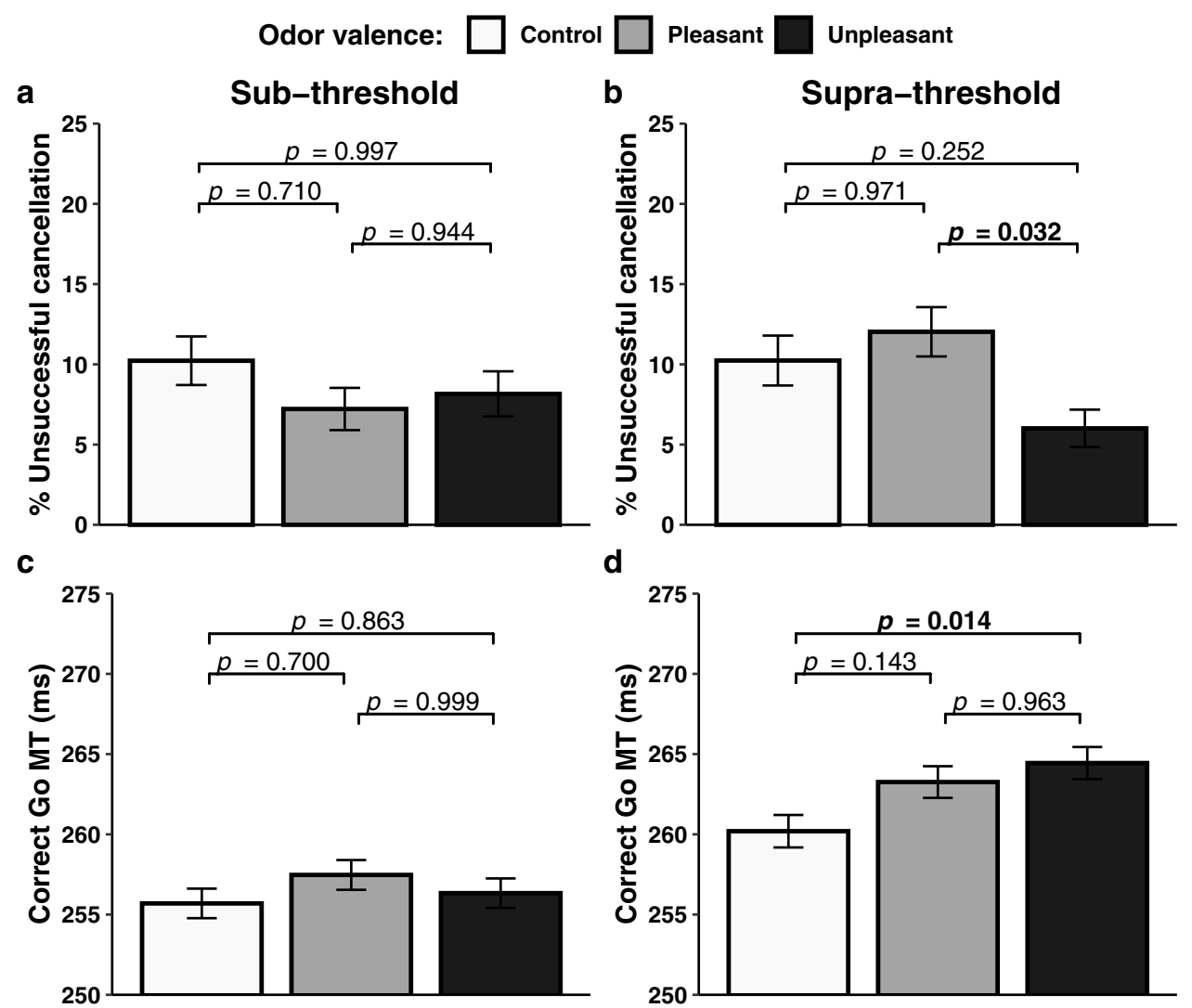
Correct Go Reaching Responses are Slower When Presented with Supra-threshold Unpleasant (vs. control) Odours

A significant main effect of odour was found on the MT of correct Go reaching responses, $\chi^{2}(2)=11.304$, $p=0.003, \mathrm{AIC}_{\mathrm{RL}}=38.547, R_{\mathrm{m}}^{2}<0.001, R^{2}{ }_{\mathrm{c}}=0.341$, with faster responses during exposure to the control odour $(258 \pm 59 \mathrm{~ms})$ as compared to both pleasant $(260 \pm 59 \mathrm{~ms}$, $p=0.018)$ and unpleasant odours $(260 \pm 59 \mathrm{~ms}, p=0.006)$, which instead did not differ significantly $(p=0.937)$. The main effect of threshold was significant too, $\chi^{2}(1)=237.42$, $p<0.001, \mathrm{AIC}_{\mathrm{RL}}>100, R_{\mathrm{m}}^{2}=0.007, R_{\mathrm{c}}^{2}=0.354$, with participants being faster in the sub-threshold condition $(256 \pm 59)$ than in the supra-threshold one $(263 \pm 60 \mathrm{~ms})$. The interaction valence $\times$ threshold was not significant, $\chi^{2}(2)=2.555, p=0.279, \mathrm{AIC}_{\mathrm{RL}}=0.485, R_{\mathrm{m}}^{2}=0.007$, $R^{2}{ }_{\mathrm{c}}=0.354$. Planned contrasts (see Fig. 3c, d) revealed slower responses in trials preceded by the unpleasant odour ( $264 \pm 60 \mathrm{~ms}$ ) in comparison to trials preceded by the control odour $(260 \pm 60 \mathrm{~ms}, p=0.014)$ in the supra-threshold condition. Other comparisons were not significant [suprathreshold: control vs. pleasant $(263 \pm 59 \mathrm{~ms}, p=0.143)$, pleasant vs. unpleasant $(p=0.963)$; sub-threshold: control $(256 \pm 58 \mathrm{~ms})$ vs. pleasant $(257 \pm 59 \mathrm{~ms}, p=0.700)$, control vs. unpleasant ( $256 \pm 58 \mathrm{~ms}, p=0.863)$, pleasant vs. unpleasant $(p=0.999)]$. In short, the MT of the reaching phase of the reach-to-press response was modulated by the presentation of odour primes. Particularly, the MT was slightly (albeit significantly) slower when the supra-threshold unpleasant odour was presented as compared to the control odour.

\section{Discussion}

In the present study, we examined the effect of pleasant (orange) and unpleasant (trimethyloxazole) odour primes presented at sub-threshold and supra-threshold levels on two forms of motor response inhibition-action withholding and action cancellation-in a modified version of the $\mathrm{Go} /$ No-Go task in which participants responded with an articulated reach-to-press action. In line with our hypotheses, for the initial release phase of the motor response, we found that the participants had more difficulties refraining from responding when a supra-threshold pleasant odour prime preceded a No-Go target as compared to the control odour (clean air), whereas supra-threshold unpleasant odours and subliminal odours did not have a significant effect on action withholding. Furthermore, when Go targets were presented, the participants made fewer omissions when exposed to the pleasant odour as compared to the control odour, regardless of its threshold. Moreover, response readiness was facilitated when sub- and supra-threshold odours preceded a Go target as compared to the control odour. In this regard, when the odours were presented at sub-threshold level, no difference was found between trials preceded by pleasant vs. unpleasant primes, while when presented at the supra-threshold level, a valence effect was revealed as the pleasant odour triggered faster responses as compared to the unpleasant one. As for the late reaching phase of the motor response, we found that cancelling an initiated inappropriate action was more challenging following a supra-threshold pleasant odour as compared to an unpleasant one, while sub-threshold odours did not have any significant effect on action cancellation. Additionally, an interference was found when the suprathreshold unpleasant odour preceded a Go target, reflected in a small, albeit significantly, delayed completion of the reaching action as compared to the control odour; such effect was not retrieved when sub-threshold odours were presented.

The present study is in line with previous findings showing that task-irrelevant pleasant odours significantly impair action withholding when presented supraliminally (Albayay et al. 2019). This is in line with the spillover theory (Chiu et al. 2014), which posits that the presentation of appetitive vs. neutral primes induces greater motor excitability, requiring greater mobilisation of inhibitory resources to withhold motor responses. Neural evidence has suggested that such interference effects are due to the prioritisation of affective stimuli of different valence, as evident by the elicitation of greater N170 amplitudes. Specifically, Yang et al. (2014) showed that emotional (vs. neutral) facial expressions elicited larger N170 amplitudes-an event-related potential (ERP) component indicating perception processing-during No-Go trials, pointing out that emotional cues have priority in trials requiring action withholding. We also found a significant modulation of action cancellation by the presentation of odour primes, but only when presented at suprathreshold level: inappropriately initiated responses were stopped less frequently when No-Go targets were primed with a pleasant (vs. unpleasant) odour. In this vein, Stockdale et al. (2019) found more negative N170 amplitude in afraid (vs. happy) facial expression in the Stop-Signal task, suggesting a prioritisation of threat-related stimuli when action cancellation must be implemented. However, our results revealed no modulation of action withholding nor of action cancellation when sub-threshold odours primed No-Go targets. In line with Verbruggen and De Houwer (2007), only high arousing emotional stimuli have been shown to interfere with response inhibition, as they attract more attentional resources away from ongoing activities. Importantly, these authors used an emotional version of the Stop-Signal paradigm, which is often used to measure action cancellation (Zhang et al. 2017).

We also extend previous results on the impact of odours primes on Go performance (Albayay et al. 2019), as both supra- and sub-threshold odours facilitated response readiness as compared to the control odour, and more accurate 
Go responses were made following the pleasant (vs. control) odour prime, regardless of its threshold. It should be noted that both the pleasant and the unpleasant sub-threshold odour stimuli triggered faster responses as compared to the control odour, but did not differ between them, as revealed in the supra-threshold condition. It is possible that the odour stimuli, which significantly differed in pleasantness when presented at supra-threshold level, were non-consciously perceived as relatively more pleasant than at their higher concentrations, thus leading to faster responses. These results support the idea that more attentional resources are allocated to emotional (vs. non-emotional) stimuli (Yang et al. 2014), resulting in a faciliatory effect on response times to visual targets (Albert et al. 2010; Chiu et al. 2014; Waring et al. 2019). However, it should be noted that only the pleasant odour elicited fewer omissions to Go as compared to the control odour in the sub-threshold condition, indicating that lapses of attention were less frequent in this condition. Similarly, Stockdale et al. (2019) and Waring et al. (2019) found that positively (vs. negatively and neutral) valenced task-irrelevant faces facilitated Go performance as reflected in more accurate Go responses. All in all, our results regarding Go performance support the bias towards approach behaviour by pleasant cues stated by the spillover theory (Chiu et al. 2014). It should be noted that the threshold test—carried out to determine subjectively determined sub-threshold concentrations-showed that the participants of this study were unable to explicitly detect the sub-threshold odour stimuli. However, the modulation of Go performance suggests a form of implicit olfactory processing of the sub-threshold odour primes, which has been previously linked to manifest changes in motor behaviour, even in participants with confirmed anosmia (Parma et al. 2013). Future research might include the recording of olfactory event-related potentials to account for the explicit and implicit processing of task-irrelevant odour primes in response inhibition paradigms.

Although the scarce literature testing the effect of subliminal emotional stimuli on motor response inhibition did not find a facilitation of Go performance (e.g. Parkinson et al. 2017), studies using non-emotional primes have shed some light in this regard. For instances, Hughes et al. (2009) found faster responses for masked Go primes as compared to No-Go and neutral primes, and more accurate Go responses following Go (vs. No-Go) primes. On the other hand, Eimer and Schlaghecken (2002) found faster RT in compatible trials (i.e. Go primes and Go targets were arrows pointing in the same direction) as compared to incompatible ones. We equate these positive compatibility effects to the notion that pleasant odours act as Go primes, as supported by the spillover theory (Chiu et al. 2014). Bocanegra and Zeelenberg (2012) extended these results by including emotional and non-emotional task-irrelevant stimuli (i.e. flanker fearful and neutral faces), and found that the presence of emotional faces increases positive (and negative) compatibility effects even when the visual primes were masked. It should be noted that in our study, all in all, the sub-threshold condition elicited faster latencies than the supra-threshold one. This could be due to a lower capture of attentional resources by the lowintensity, less distractive primes. In a similar vein, the latencies of the reaching phase of the motor response (MT) were modulated only by supra-threshold odours, with slightly slower responses when unpleasant primes were presented as compared to the control condition. We hypothesised that this was due to the effect of unpleasant stimuli acting as Stop primes, which relates to the interference effect found by Ocampo et al. (2015) for incongruent primes, only that they revealed this effect using non-consciously perceived visual primes.

It should be pointed out that although previous studies have addressed the impact of task-irrelevant affective stimuli valence (e.g. Albayay et al. 2019; Chiu et al. 2014; Waring et al. 2019) and arousal (e.g. Pearlstein et al. 2019; Pessoa et al. 2012) on response inhibition, those studies that directly contrasted both accounts (e.g. Albert et al. 2010; Verbruggen and De Houwer 2007; Zhao et al. 2019) have yielded inconsistent results. For instance, Verbruggen and De Houwer (2007) included low and high arousing visual stimuli (human faces) of different valence (positive, negative and neutral) as primes in a Stop-Signal task. It was revealed that only high arousing affective stimuli impaired response inhibition, as reflected in longer Stop-Signal reaction times-an index of the covert latency of response inhibition-whereas valence had little influence. Conversely, in a Go/No-Go task Albert et al. (2010) found that No-Go P3 amplitude - an ERP component recruited when response inhibition is required - was larger when presented with positive (vs. negative) faces. No-Go P3 amplitude increased as the affective stimuli were more positive but did not correlate with affective arousal. Further, the authors found no modulation of behavioural No-Go performance by the emotional stimuli. More recently, Zhao et al. (2019) found smaller No-Go N2-an ERP component associated with conflict monitoring-and larger No-Go P3 amplitudes when presented with positive and negative (vs. neutral) visual stimuli. It was suggested that response inhibition is modulated by the affected arousal of task-irrelevant stimuli, while response readiness is affected by affective valence. A critical aspect is that all the aforementioned studies used visual affective primes in their designs. Our results highlight the limitations of assuming that all sensory information at different levels of awareness equally affect response inhibition. It should be pointed out that although we measured the perceived intensity of the odour primes as a proxy for arousal (Bensafi et al. 2002; Cecchetto et al. 2017), we did not include any explicit measure of emotional arousal. Futures studies should include 
objective real-time arousal assessments-such as skin conductance response and electroencephalographic activationto account for the participants' arousal level at the moment of smelling the odour stimuli.

Overall, our results revealed that both valence and threshold of emotional stimuli are key elements in the modulation of response inhibition and response readiness when it comes to odour cues. Valence influences on inhibitory performance (action withholding and action cancellation) were revealed only when the odours smelled were more arousing (i.e. supra-threshold). Instead, response readiness was facilitated even when the odours were less arousing (i.e. sub-threshold), suggesting that although not consciously perceived, the odour primes could have been more arousing than the control condition.

\section{Conclusions}

All in all, our results contribute to the literature on the effect of odour stimuli on goal-directed behaviour. By using odours, we were able to uncover the boundaries of the response inhibition processes (from readiness to cancellation) across different levels of pleasantness and threshold in a modified Go/No-Go task. The important aspect of the present findings is the characterisation of the impact of chemosensory stimuli on response inhibition processeswhich has been extensively studied in the visual domainhighlighting the interaction of the valence and the threshold level of odour cues in the modulation of response readiness, action withholding and action cancellation. What emerges is that when the odour stimulus is pleasant and consciously perceived, reach-to-press actions are less inhibited both at the release (withholding) and at the reaching (cancellation) phases.

It is important to note though that the odours presented here were neither easy to label and have elicited a differential conscious representation that could have driven the action, nor were associated with the visual target (a circle or a square) or the action performed. We believe that this link will be significant in modifying the ability to withhold and cancel actions which are not only salient (like supra threshold elicited actions here) but also relevant to the context. Future studies are left to understand these aspects and to evaluate possible applications of binge drinking and eating scenarios. The introduction of this pleasantness uncertainty, which is uniquely offered by the use of odours as compared to the subliminal presentation of facial expressions, helps to map the continuum of response inhibition. Although here we argue that valence is a crucial aspect in the modulation of response inhibition by odour primes, we did not measure how emotionally laden these stimuli were for the participants. Future studies should consider individual differences in this regard, given that not all odours are equally emotionally laden for all individuals. Future research should also consider odour edibility as a possible factor affecting response inhibition. Moreover, it should be noted that most odorous substances at high enough concentrations activate not only the olfactory nerve but also the trigeminal nerve (Doty et al. 1978; Frasnelli et al. 2011). Although we have not collected measures of trigeminality for the odours used in this study, the analysis of the self-reports from this study and previous research (Albayay et al. 2019) suggests that trimethyloxazole has been reported by some participants as somewhat irritating. Indeed, the pleasantness ratings for trimethyloxazole (but not orange) are compatible with the presence of trigeminal stimulation in the suprathreshold conditions. It is worth noting however that intensity ratings were similar for the trimethyloxazole and orange odour in the supra-threshold session.

The present results complement the literature on reach-tograsp interference effects by demonstrating that the mechanisms of selection for the control of an overt hand action take into account irrelevant olfactory information (e.g. Castiello et al. 2006). Although in this study, we attempted to extend the impact of odour cues on more articulated motor responses (reach-to-press) as compared to previous studies focusing on simple keypress actions including chemosensory stimuli (e.g. Albayay et al. 2019; Chiu et al. 2014), our design is not free of limitations. Future studies should address more direct comparisons between simple and complex motor action by including and alternating different responses in the same experimental task (e.g. alternate response type base on visual cues; Gálvez-García et al. 2018). Moreover, despite our results on the impact of emotional stimuli on action cancellation are in line with previous results in the Stop-Signal paradigm (i.e. high arousing stimuli interfering with action cancellation; Verbruggen and De Houwer 2007), future studies should explore whether the modification of the Go/No-Go paradigm to account for action cancellation yields to comparable results in the StopSignal paradigm, as it has been shown that both tasks involve different inhibitory mechanisms (Littman and Takács 2017; Raud et al. 2020; Zhang et al. 2017).

Acknowledgements We would like to the SISSA Mechatronics Lab and Dr. Stefano Massaccesi for their technical support. We also thank Mariagiovanna Berardo for her help with data acquisition.

Authors' Contributions All authors contributed to the study conception and design. Material preparation, data collection, and analysis were performed by Javier Albayay and Valentina Parma. All authors contributed to the first draft of the manuscript.

Funding Open access funding provided by Università degli Studi di Padova within the CRUI-CARE Agreement. This work was supported by a scholarship from the National Research and Development Agency (ANID, Chile) PFCHA/DOCTORADO BECAS CHILE/2017 
[72180384] granted to Javier Albayay, and by the research program Dipartimenti di Eccellenza (art.1, commi 314-337 legge 232/2016), which was supported by a grant from Ministry of Education, University and Research (MIUR, Italy) to the Department of General Psychology, University of Padova.

Availability of Data and Materials The data and R scripts generated during the current study are available in the Open Science Framework repository accessible at https://osf.io/3jt6s/?view_only=d6b37915ec $66498 \mathrm{a} 8 \mathrm{dc} 86 \mathrm{f} 55 \mathrm{c} 797 \mathrm{a} 35 \mathrm{f}$

\section{Declarations}

Ethics Approval and Consent to Participate All participants gave written informed consent and did not receive any monetary compensation. The study was approved by the local Institutional Review Board (International School for Advanced Studies, Trieste, Italy) and was conducted in accordance with the Declaration of Helsinki and the ethical standards of the American Psychological Association.

Competing Interests Non-financial interests: Valentina Parma is a scientific consultant for CyNexo. All other authors declare that they have no conflict of interest.

Open Access This article is licensed under a Creative Commons Attribution 4.0 International License, which permits use, sharing, adaptation, distribution and reproduction in any medium or format, as long as you give appropriate credit to the original author(s) and the source, provide a link to the Creative Commons licence, and indicate if changes were made. The images or other third party material in this article are included in the article's Creative Commons licence, unless indicated otherwise in a credit line to the material. If material is not included in the article's Creative Commons licence and your intended use is not permitted by statutory regulation or exceeds the permitted use, you will need to obtain permission directly from the copyright holder. To view a copy of this licence, visit http://creativecommons.org/licenses/by/4.0/.

\section{References}

Adolph D, Pause BM (2012) Different time course of emotion regulation towards odors and pictures: Are odors more potent than pictures? Biol Psychol 91:65-73. https://doi.org/10.1016/j.biops ycho.2012.05.003

Albayay J, Castiello U, Parma V (2019) Task-irrelevant odours affect both response inhibition and response readiness in fast-paced Go/No-Go task: the case of valence. Sci Rep 9:19329. https:// doi.org/10.1038/s41598-019-55977-z

Albert J, López-Martín S, Carretié L (2010) Emotional context modulates response inhibition: neural and behavioral data. Neuroimage 49:914-921. https://doi.org/10.1016/j.neuroimage.2009. 08.045

American Psychological Association (2017) Ethical Principles of Psychologists and Code of Conduct. Retrieved from https://www.apa. org/ethics/code/

Angelini M, Calbi M, Ferrari A, Sbriscia-Fioretti B, Franca M, Gallese V, Umiltà MA (2015) Motor inhibition during overt and covert actions: an electrical neuroimaging study. PLoS ONE 10:e0126800. https://doi.org/10.1371/journal.pone.0126800

Angelini M, Calbi M, Ferrari A, Sbriscia-Fioretti B, Franca M, Gallese V, Umiltà MA (2016) Proactive control strategies for overt and covert go/nogo tasks: an electrical neuroimaging study. PLoS ONE 11:e0155007. https://doi.org/10.1371/journal.pone.0155007

Aron AR (2011) From reactive to proactive and selective control: developing a richer model for stopping inappropriate responses. Biol Psychiat 69:e55-e68. https://doi.org/10.1016/j.biopsych. 2010.07.024

Bates D, Maechler M, Bolker B et al (2018) lme4: linear mixedeffects models using 'Eigen' and S4 (R package version 1.1-17). Retrieved from https://cran.r-project.org/web/packages/lme4/ index.html

Beck AT, Steer RA, Brown GK (1996) Manual for the Beck Depression Inventory-II. Psychological Corporation, San Antonio

Bensafi M, Rouby C, Farget V, Bertrand B, Vigouroux M, Holley A (2002) Autonomic nervous system responses to odours: the role of pleasantness and arousal. Chem Senses 27:703-709. https:// doi.org/10.1093/chemse/27.8.703

Bocanegra BR, Zeelenberg R (2012) Emotion potentiates response activation and inhibition in masked priming. Front Integr Neurosci 6:109. https://doi.org/10.3389/fnint.2012.00109

Boy F, Clarke K, Sumner P (2008) Mask stimulus triggers inhibition in subliminal visuomotor priming. Exp Brain Res 190:111-116. https://doi.org/10.1007/s00221-008-1515-5

Castiello U, Zucco GM, Parma V, Ansuini C, Tirindelli R (2006) Crossmodal interactions between olfaction and vision when grasping. Chem Senses 31:665-671. https://doi.org/10.1093/chemse/bj1007

Cecchetto C, Rumiati RI, Aiello M (2017) Alexithymia and emotional reactions to odors. Sci Rep 7:14097. https://doi.org/10.1038/ s41598-017-14404-x

Chen Z, Saunders JA (2018) Volitional and automatic control of the hand when reaching to grasp objects. J Exp Psychol Hum Percept Perform 44:953-972. https://doi.org/10.1037/xhp0000492

Chiu YC, Aron AR (2014) Unconsciously triggered response inhibition requires an executive setting. J Exp Psychol Gen 143:56-61. https://doi.org/10.1037/a0031497

Chiu YC, Cools R, Aron AR (2014) Opposing effects of appetitive and aversive cues on go/no-go behavior and motor excitability. J Cogn Neurosci 26:1851-1860. https://doi.org/10.1162/jocn_a_00585

Croy I, Krone F, Walker S, Hummel T (2015) Olfactory processing: detection of rapid changes. Chem Senses 40:351-355. https://doi. org/10.1093/chemse/bjv020

Diamond A (2013) Executive functions. Annu Rev Psychol 64:135168. https://doi.org/10.1146/annurev-psych-113011-143750

Donders FC (1969) On the speed of mental processes. Acta Psychol (amst) 30:412-431. https://doi.org/10.1016/0001-6918(69) 90065-1

Doty RL, Brugger WPE, Jurs PC, Orndorff MA, Snyder PJ, Lowry LD (1978) Intranasal trigeminal stimulation from odorous volatiles: psychometric responses from anosmic and normal humans. Physiol Behav 20:175-185. https://doi.org/10.1016/0031-9384(78) 90070-7

Eimer M, Schlaghecken F (2002) Links between conscious awareness and response inhibition: Evidence from masked priming. Psychon Bull Rev 9:514-520. https://doi.org/10.3758/bf03196307

Faul F, Erdfelder E, Lang AG, Buchner A (2007) G*Power 3: a flexible statistical power analysis program for the social, behavioral, and biomedical sciences. Behav Res Methods 39:175-191. https://doi. org/10.3758/bf03193146

Frasnelli J, Albrecht J, Bryant B, Lundström JN (2011) Perception of specific trigeminal chemosensory agonists. Neuroscience 189:377-383. https://doi.org/10.1016/j.neuroscience.2011.04.065

Gálvez-García G, Albayay J, Rehbein L, Bascour-Sandoval C, Michael GA (2018) Response inhibition as a function of movement complexity and movement type selection. Front Psychol 9:2290. https://doi.org/10.3389/fpsyg.2018.02290

Gottfried JA, Deichmann R, Winston JS, Dolan RJ (2002) Functional heterogeneity in human olfactory cortex: an event-related 
functional magnetic resonance imaging study. J Neurosci 22:10819-10828. https://doi.org/10.1523/JNEUROSCI.22-2410819.2002

Grös DF, Antony MM, Simms LJ, McCabe RE (2007) Psychometric properties of the State-Trait Inventory for Cognitive and Somatic Anxiety (STICSA): comparison to the State-Trait Anxiety Inventory (STAI). Psychol Assess 19:369-381. https://doi.org/10.1037/ 1040-3590.19.4.369

Hughes G, Velmans M, De Fockert J (2009) Unconscious priming of a no-go response. Psychophysiology 46:1258-1269. https://doi. org/10.1111/j.1469-8986.2009.00873.x

Hummel T, Sekinger B, Wolf SR, Pauli E, Kobal G (1997) "Sniffin" sticks': olfactory performance assessed by the combined testing of odor identification, odor discrimination and olfactory threshold. Chem Senses 22:39-52. https://doi.org/10.1093/chemse/22.1.39

Hummel T, Kobal G, Gudziol H, Mackay-Sim A (2007) Normative data for the "Sniffin' Sticks" including tests of odor identification, odor discrimination, and olfactory thresholds: an upgrade based on a group of more than 3,000 subjects. Eur Arch Otorhinolaryngol 264:237-243. https://doi.org/10.1007/ s00405-006-0173-0

Lefcheck JS (2016) PiecewiseSEM: Piecewise structural equation modelling in $r$ for ecology, evolution, and systematics. Methods Ecol Evol 7:573-579. https://doi.org/10.1111/2041-210X.12512

Lenth RV (2016) Least-squares means: the R package lsmeans. J Stat Softw 69:1-33. https://doi.org/10.18637/jss.v069.i01

Li W (2014) Learning to smell danger: acquired associative representation of threat in the olfactory cortex. Front Behav Neurosci 8:98. https://doi.org/10.3389/fnbeh.2014.00098

Li W, Moallem I, Paller KA, Gottfried JA (2007) Subliminal smells can guide social preferences. Psychol Sci 18:1044-1049. https:// doi.org/10.1111/j.1467-9280.2007.02023.x

Littman R, Takács Á (2017) Do all inhibitions act alike? A study of go/no-go and stop-signal paradigms. PLoS ONE 12:e0186774. https://doi.org/10.1371/journal.pone.0186774

McElreath R (2016) Statistical rethinking: A bayesian course with examples in R and Stan. CRC Press, Boca Raton

Moskowitz HR, Dravnieks A, Klarman LA (1976) Odor intensity and pleasantness for a diverse set of odorants. Atten Percept Psychophys 19:122-128. https://doi.org/10.3758/BF03204218

Nguyen AT, Moyle JJ, Fox AM (2016) N2 and P3 modulation during partial inhibition in a modified go/nogo task. Int J Psychophysiol 107:63-71. https://doi.org/10.1016/j.ijpsycho.2016.07.002

Ocampo B, Finkbeiner M (2013) The negative compatibility effect with relevant masks: a case for automatic motor inhibition. Front Psychol 4:822. https://doi.org/10.3389/fpsyg.2013.00822

Ocampo B, Al-Janabi S, Finkbeiner M (2015) Direct evidence of cognitive control without perceptual awareness. Psychon Bull Rev 22:1083-1088. https://doi.org/10.3758/s13423-014-0766-3

Oldfield RC (1971) The assessment and analysis of handedness: the Edinburgh Inventory. Neuropsychologia 9:97-113. https://doi. org/10.1016/0028-3932(71)90067-4

Parkinson J, Haggard P (2014) Subliminal priming of intentional inhibition. Cognition 130:255-265. https://doi.org/10.1016/j. cognition.2013.11.005

Parkinson J, Garfinkel S, Critchley H, Dienes Z, Seth AK (2017) Don't make me angry, you wouldn't like me when I'm angry: volitional choices to act or inhibit are modulated by subliminal perception of emotional faces. Cogn Affect Behav Neurosci 17:252-268. https://doi.org/10.3758/s13415-016-0477-5

Parma V, Bulgheroni M, Scaravilli T, Tirindelli R, Castiello U (2013) Implicit olfactory processing attenuates motor disturbances in idiopathic Parkinson's disease. Cortex 49:1241-1251. https:// doi.org/10.1016/j.cortex.2012.05.017

Parma V, Ferraro S, Miller SS, Åhs F, Lundström JN (2015) Enhancement of odor sensitivity following repeated odor and visual fear conditioning. Chem Senses 40:497-506. https://doi. org/10.1093/chemse/bjv033

Pearlstein JG, Johnson SL, Modavi K, Peckham AD, Carver CS (2019) Neurocognitive mechanisms of emotion-related impulsivity: The role of arousal. Psychophysiology 56:e13293. https://doi.org/10.1111/psyp.13293

Pessoa L, Padmala S, Kenzer A, Bauer A (2012) Interactions between cognition and emotion during response inhibition. Emotion 12:192-197. https://doi.org/10.1037/a0024109

Pinheiro J, Bates D, DebRoy S, Sarkar D, R Core Team (2020) nlme: Linear and nonlinear mixed effects models (R package version 3.1-148). Retrieved from https://CRAN.R-project.org/packa ge $=$ nlme

Psychology Software Tools, Inc (2012) E-Prime 2.0. Available at http://www.pstnet.com

Raud L, Westerhausen R, Dooley N, Huster RJ (2020) Differences in unity: The go/no-go and stop signal tasks rely on different mechanisms. Neuroimage 210:116582. https://doi.org/10. 1016/j.neuroimage.2020.116582

Rouby C, Pouliot S, Bensafi M (2009) Odor hedonics and their modulators. Food Qual Prefer 20:545-549. https://doi.org/10.1016/j. foodqual.2009.05.004

RStudio Team (2016) RStudio: integrated development for R. RStudio, Inc., Boston, MA. Retrieved from http://www.rstudio.com/

Singmann H, Kellen D (2017) An introduction to mixed models for experimental psychology. In: Spieler DH, Schumacher E (eds) New Methods in Cognitive Psychology. Psychology Press, New York, pp 4-31

Smeets MA, Dijksterhuis GB (2014) Smelly primes - when olfactory primes do or do not work. Front Psychol 5:96. https://doi.org/ 10.3389/fpsyg. 2014.00096

Stockdale LA, Morrison RG, Silton RL (2019) The influence of stimulus valence on perceptual processing of facial expressions and subsequent response inhibition. Psychophysiology 57:e13467. https://doi.org/10.1111/psyp.13467

Sumner P (2007) Negative and positive masked-priming - implications for motor inhibition. Adv Cogn Psychol 3:317-326. https://doi.org/10.2478/v10053-008-0033-0

van Gaal S, Ridderinkhof KR, van den Wildenberg WPM, Lamme VAF (2009) Dissociating consciousness from inhibitory control: evidence for unconsciously triggered response inhibition in the stop-signal task. J Exp Psychol Hum Percept Perform 35:1129-1139. https://doi.org/10.1037/a0013551

van Gaal S, Ridderinkhof KR, Scholte HS, Lamme VAF (2010) Unconscious Activation of the Prefrontal No-Go Network. J Neurosci 30:4143-4150. https://doi.org/10.1523/JNEUROSCI. 2992-09.2010

Verbruggen F, De Houwer J (2007) Do emotional stimuli interfere with response inhibition? Evidence from the stop signal paradigm. Cogn Emot 21:391-403. https://doi.org/10.1080/02699 930600625081

Verbruggen F, Logan GD (2017) Control in response inhibition. In: Egner T (ed) The Wiley Handbook of Cognitive Control. John Wiley \& Sons, Oxford, pp 97-110

Vieno A, Altoè G, Kuntsche E, Elgar FJ (2018) Do public expenditures on health and families relate to alcohol abstaining in adolescents? Multilevel study of adolescents in 24 countries. Drug Alcohol Rev 37:S120-S128. https://doi.org/10.1111/dar.12696

Waring JD, Greif TR, Lenze EJ (2019) Emotional response inhibition is greater in older than younger adults. Front Psychol 10:961. https://doi.org/10.3389/fpsyg.2019.00961

Wessel JR (2017) Prepotent motor activity and inhibitory control demands in different variants of the go/no-go paradigm. Psychophysiology 55:e12871. https://doi.org/10.1111/psyp.12871 
Wilson DA, Stevenson RJ (2006) Learning to smell: olfactory perception from neurobiology to behavior. Johns Hopkins University Press, Baltimore

World Medical Association (2013) WMA Declaration of Helsinki: Ethical principles for medical research involving human subjects. JAMA 310:2191-2194. https://doi.org/10.1001/jama. 2013.281053

Yang S, Luo W, Zhu X, Broster LS, Chen T, Li J, Luo Y (2014) Emotional content modulates response inhibition and perceptual processing. Psychophysiology 51:1139-1146. https://doi.org/ 10.1111/psyp. 12255

Zamorano F, Billeke P, Hurtado JM, López V, Carrasco X, Ossandón T, Aboitiz F (2014) Temporal constraints of behavioral inhibition: relevance of inter-stimulus interval in a go-nogo task. PLoS ONE 9:e87232. https://doi.org/10.1371/journal.pone. 0087232
Zhang R, Geng X, Lee TMC (2017) Large-scale functional neural network correlates of response inhibition: an fMRI meta-analysis. Brain Struct Funct 222:3973-3990. https://doi.org/10.1007/ s00429-017-1443-x

Zhao D, Lin H, Xie S, Liu Z (2019) Emotional arousal elicited by irrelevant stimuli affects event-related potentials (ERPs) during response inhibition. Physiol Behav 206:134-142. https://doi.org/ 10.1016/j.physbeh.2019.04.005

Publisher's Note Springer Nature remains neutral with regard to jurisdictional claims in published maps and institutional affiliations. 